\title{
Supplemental Information for Surface Shear Viscosity and Interleaflet Friction from Nonequilibrium Simulations of Lipid Bilayers
}

Andrew Zgorski, ${ }^{\dagger}$ Richard W. Pastor, ${ }^{\ddagger}$ and Edward Lyman ${ }^{\S}{ }^{*}$

†Department of Physics and Astronomy, University of Delaware, Newark, 19716 Delaware, United States

Laboratory of Computational Biology, National Heart, Lung, and Blood Institute, National Institutes of Health, Bethesda Maryland 20892, United States

$\S$ Department of Physics and Astronomy and Department of Chemistry and Biochemistry, University of Delaware, Newark, 19716 Delaware, United States

*Corresponding author: elyman@udel.edu

\section{Supplemental Methods}

Table S1: Simulation parameters for Martini water in GROMACS.

\begin{tabular}{|l|l|}
\hline Integration & $\begin{array}{l}\text { Velocity-Verlet with } \Delta t=20 \text { fsec } \\
\text { Center of mass motion removed every timestep }\end{array}$ \\
\hline Neighbor Lists & $\begin{array}{l}\text { Group scheme, recalculated every } 10 \text { timesteps; } \\
\text { Cutoff at } 14 \AA\end{array}$ \\
\hline Nonbonded & $\begin{array}{l}\text { Van der Waals Potential shifted to zero over } 9-12 \AA . \\
\text { Electrostatic potential shifted to zero over } 0-12 \AA ; \\
\text { No long-range electrostatics; } \\
\text { Relative permittivity } \varepsilon_{r}=15\end{array}$ \\
\hline Thermostat & $\begin{array}{l}\text { Bussi velocity rescaling; } \\
T_{0}=323 \mathrm{~K}, \tau_{T}=1 \text { psec }\end{array}$ \\
\hline Barostat & $\begin{array}{l}\text { Berendsen, isotropic coupling; } \\
P_{0}=1 \text { bar, } \tau_{P}=12 \text { psec } \\
\text { compressibility }=3.0 \times 10^{-4} \text { bar }{ }^{-1}\end{array}$ \\
\hline
\end{tabular}


Table S2: Simulation parameters for TIP3P water with CHARMM36 in GROMACS.

\begin{tabular}{|l|l|}
\hline Integration & $\begin{array}{l}\text { Velocity-Verlet with } \Delta t=2 \text { fsec } \\
\text { Center of mass motion removed every } 100 \text { timesteps, treating } \\
\text { membrane and solvent separately }\end{array}$ \\
\hline Neighbor Lists & $\begin{array}{l}\text { Verlet scheme, recalculated every } 10 \text { timesteps; } \\
\text { Cutoff at } 12 \AA\end{array}$ \\
\hline Nonbonded & $\begin{array}{l}\text { Force switched to zero over } 8 \text {-12 } \AA \\
\text { PME used for long-range electrostatics }\end{array}$ \\
\hline Bonded & $\begin{array}{l}\text { All hydrogen bonds constrained with LINCS algorithm } \\
\text { Thermostat }\end{array}$ \\
\hline Barostat & $\begin{array}{l}T_{0}=323 \mathrm{~K}, \tau_{T}=1 \text { psec } \\
P_{0}=1 \text { bar, } \tau_{P}=5 \text { psec } \\
\text { compressibility }=4.5 \times 10^{-5} \text { bar }{ }^{-1}\end{array}$ \\
\hline
\end{tabular}


Table S3: Simulation parameters for Martini membranes in GROMACS.

\begin{tabular}{|l|l|}
\hline Integration & $\begin{array}{l}\text { Velocity-Verlet with } \Delta t=20 \text { fsec } \\
\text { Center of mass motion removed every timestep, treating } \\
\text { membrane and solvent separately }\end{array}$ \\
\hline Neighbor Lists & $\begin{array}{l}\text { Group scheme, recalculated every } 10 \text { timesteps; } \\
\text { Cutoff at } 14 \AA\end{array}$ \\
\hline Nonbonded & $\begin{array}{l}\text { Potential shifted to zero over } 9-12 \AA . \\
\text { Electrostatic potential shifted to zero over } 0-12 \AA ; \\
\text { No long-range electrostatics; } \\
\text { Relative permittivity } \varepsilon_{r}=15\end{array}$ \\
\hline Thermostat & $\begin{array}{l}\text { Bussi velocity rescaling, treating water and lipids separately; } \\
T_{0}=323 \mathrm{~K}, \tau_{T}=1 \text { psec } \\
\text { Berendsen, semiisotropic coupling; } \\
P_{0}=0 \text { bar, } \tau_{P}=12 \text { psec } \\
\text { Compressibility }=3.0 \times 10^{-4} \text { bar }{ }^{-1} \text { (lateral, }=0 \text { for normal) }\end{array}$ \\
\hline Barostat &
\end{tabular}


Table S4: Simulation parameters for CHARMM36 membranes in GROMACS.

\begin{tabular}{|c|c|}
\hline Integration & $\begin{array}{l}\text { Leapfrog with } \Delta t=2 \mathrm{fsec} \\
\text { Center of mass motion removed every } 100 \text { timesteps, treating } \\
\text { membrane and solvent separately. }\end{array}$ \\
\hline Neighbor Lists & $\begin{array}{l}\text { Verlet scheme, recalculated every } 10 \text { timesteps; } \\
\text { Cutoff at } 12 \AA\end{array}$ \\
\hline Nonbonded & $\begin{array}{l}\text { Force switched to zero over } 8-10 \text { or 8-12 } \AA \text {. } \\
\text { PME used for long-range electrostatics }\end{array}$ \\
\hline Bonded & All hydrogen bonds constrained with LINCS algorithm \\
\hline Thermostat & $\begin{array}{l}\text { Nosé-Hoover, treating water and lipids separately; } \\
T_{0}=323 \mathrm{~K}, \tau_{T}=1 \mathrm{psec}\end{array}$ \\
\hline Barostat & $\begin{array}{l}\text { Parrinello-Rahman, semiisotropic coupling; } \\
P_{0}=0 \text { bar, } \tau_{P}=5 \text { psec } \\
\text { Compressibility }=4.5 \times 10^{-5} \mathrm{bar}^{-1} \text { (lateral, }=0 \text { for normal) }\end{array}$ \\
\hline
\end{tabular}




\section{Supplemental Results}

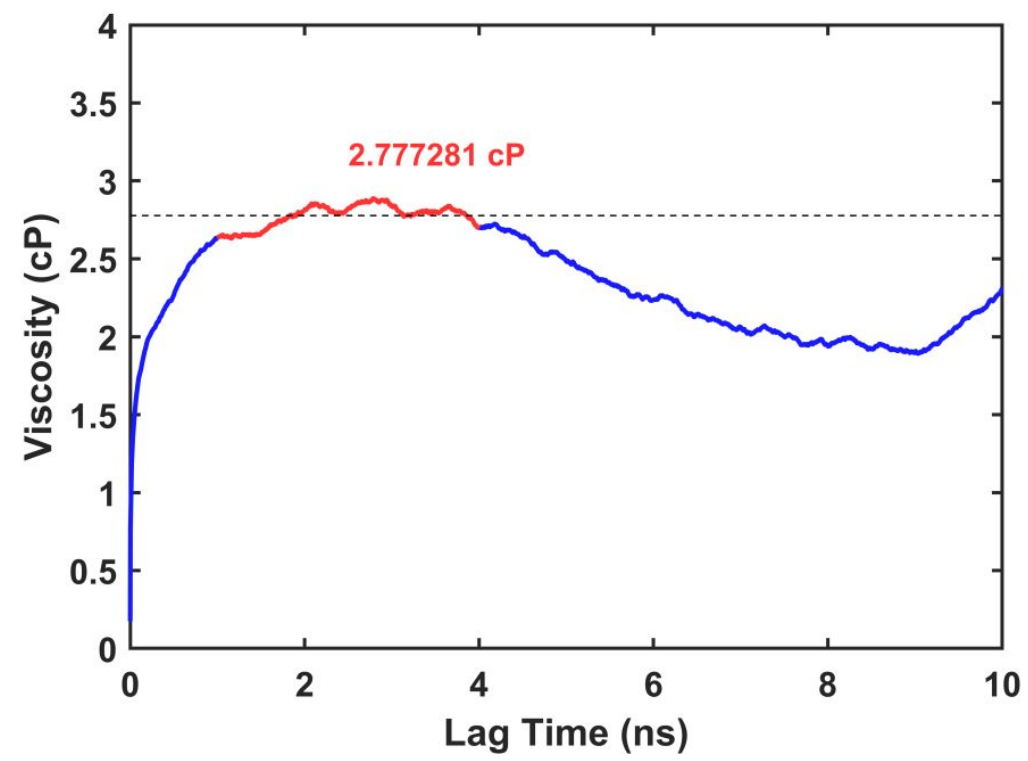

Figure S1: Sample depiction of total viscosity for Martini DPPC using the Green-Kubo relation (Equation 7). The correlation builds until a plateau is reached. The plateau is not maintained as lag times increase due to the finite length of the simulation and the decreasing number of samples at longer lag times.

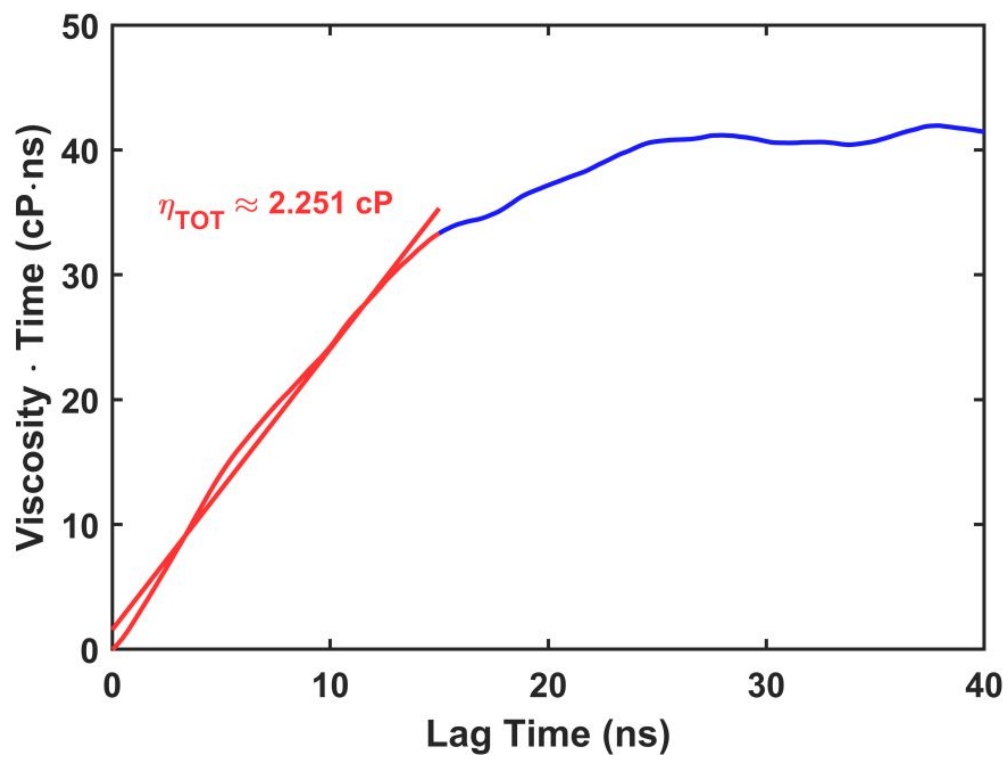

Figure S2: Sample depiction of total viscosity for Martini DPPC using the Green-Kubo equation recast as an Einstein relation (Equation 8). The expression under the derivative is plotted as a function of time. A linear fit to the linear regime gives total viscosity. This occurs for times that are long compared to the pressure fluctuations but short compared to the length of the simulation. 


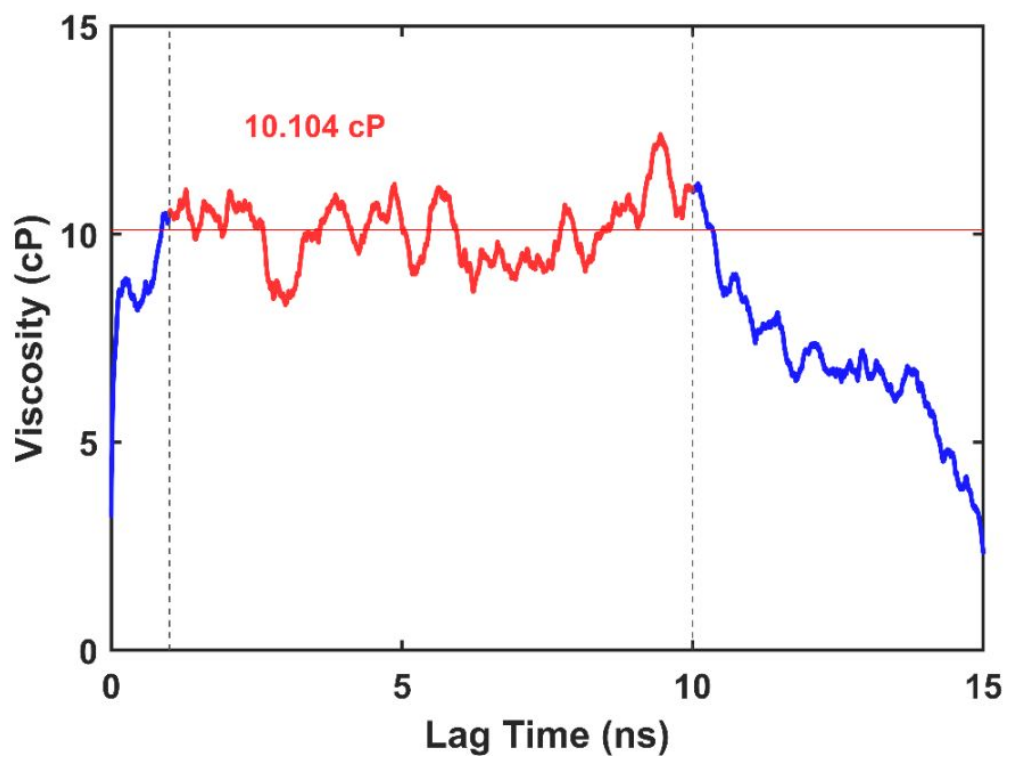

Figure S3: Sample depiction of total viscosity for CHARMM36 DPPC with 8-12 force switching using the Green-Kubo relation (Equation 7). The correlation builds until a plateau is reached. The plateau is not maintained as lag times increase due to the finite length of the simulation and the decrease in the number of samples between longer lag times.

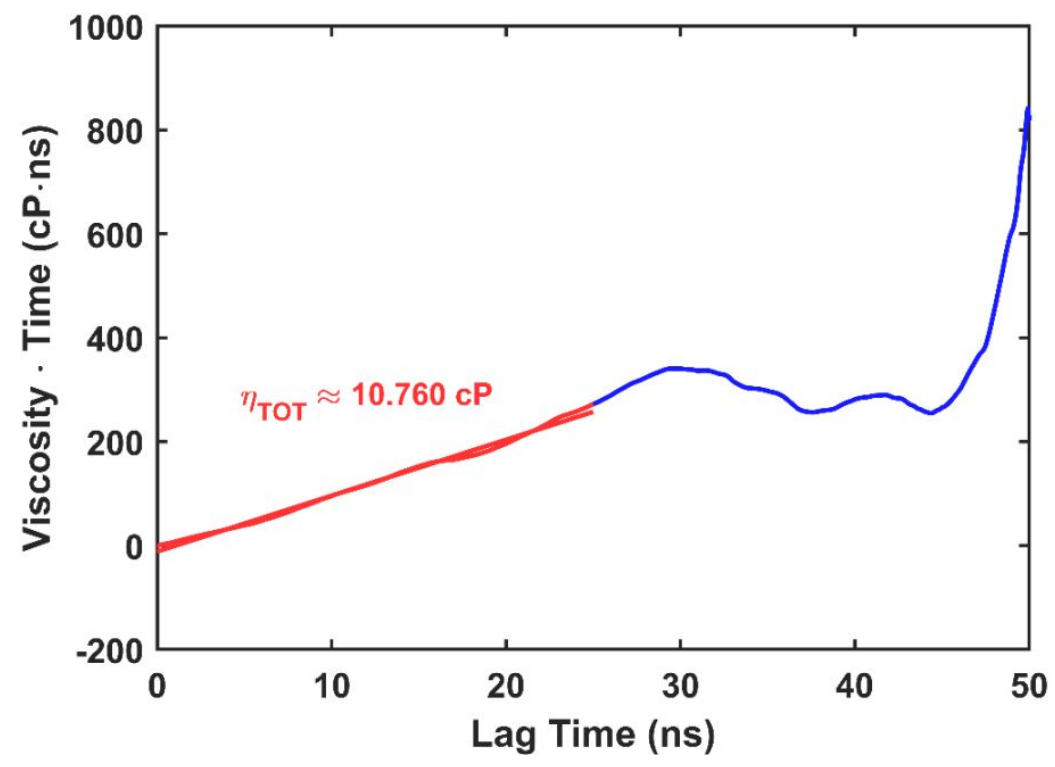

Figure S4: Sample depiction of total viscosity for CHARMM36 DPPC with 8-12 force switching using the Green-Kubo equation recast as an Einstein relation (Equation 8). The expression under the derivative is plotted as a function of time. A linear fit to the linear regime gives total viscosity. This occurs for times that are long compared to the pressure fluctuations but short compared to the length of the simulation. 


\section{Equilibrium Viscosity Calculations for C36 DPPC}

The surface viscosity results for DPPC were independently corroborated with equilibrium calculations of the total system viscosity. Five $50 \mathrm{~ns}$ replicas with random initial velocities were simulated for both force switching ranges in the NPT ensemble using the simulation parameters from Table $\mathbf{S} 4$ with the barostat disabled. Equilibrium surface viscosity was calculated using both the Green-Kubo relation from Equation 7 (Fig. S3) and its equivalent Einstein relation from Equation 8 (Fig. S4). Results for the average of each five trials are reported in Table S5, finding good agreement with values calculated from the shearing simulations, especially when $8-12 \AA$ force switching is employed. This agreement strongly suggests the deformation rates of our shearing simulations are modest enough to calculate the equilibrium surface viscosity for CHARMM36 membranes.

Table S5: Total viscosity calculated for CHARMM36 DPPC membranes. Results from shearing simulations are presented along with average equilibrium calculations for both 8-10 and 8-12 force switching.

\begin{tabular}{|l|l|}
\hline Method & $\begin{array}{l}\text { Total Viscosity } \\
\left(10^{-11} \mathrm{~Pa}-\mathrm{m}-\mathrm{s}\right)=\left(10^{-8} \mathrm{P}-\mathrm{cm}\right)\end{array}$ \\
\hline Shearing (8-10) & $8.27 \pm 0.50$ \\
\hline Einstein Relation (8-10) & $11.1 \pm 2.4$ \\
\hline Shearing (8-12) & $12.35 \pm 0.50$ \\
\hline Einstein Relation (8-12) & $12.2 \pm 1.4$ \\
\hline
\end{tabular}




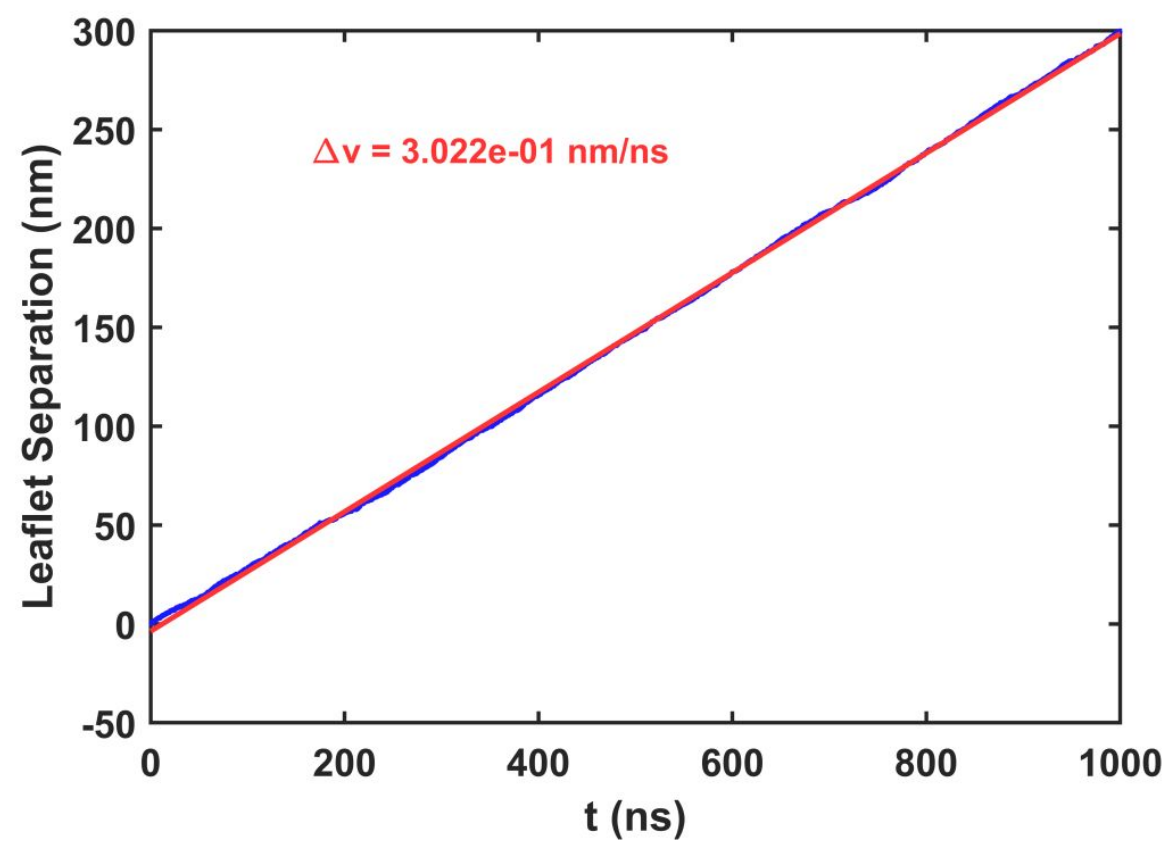

Figure S5: Distance between average leaflet positions as a function of time when the solvent exerts sufficient traction forces on the leaflets. Leaflets slide past one another at a relatively smooth, steady rate. The linear fit (red) gives their average relative velocity.

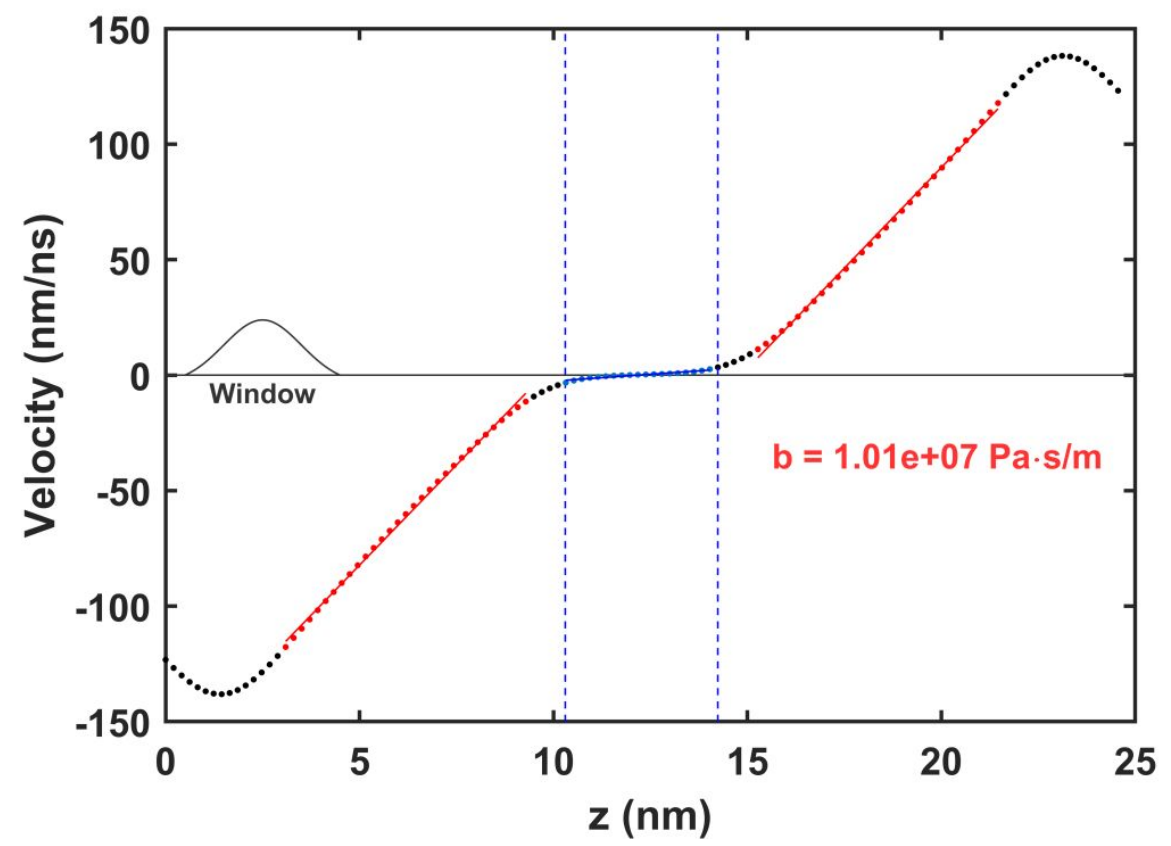

Figure S6: Average velocity profile for CHARMM36 DPPC with a $0.5 \mathrm{~kJ} /(\mathrm{mol}-\mathrm{nm})$ force applied to all particles within $1 \mathrm{~nm}$ of the boundary at every timestep. The region corresponding to the membrane is highlighted in blue and the extent of linear parallel shear flow in the solvent is highlighted in red. Fits to these regions are used to determine the solvent shear rates. The spatial averaging window used to create the velocity profile is depicted on the left. 